\title{
A Study of Surgical Outcomes of Tympanoplasties with and without Cortical Mastoidectomy
}

\section{Rajiv Kumar saxsena ${ }^{1}$, Hemendra bamaniya *2.}

${ }^{1}$ Associate Professor, Department of Otolaryngology, Ananta Institute of Medical science, rajsamand, Rajasthan, India.

*2 Assistant Professor, Department of Otolaryngology, Ananta Institute of M edical science, rajsamand, Rajasthan, India.

\section{ABSTRACT}

Background: Tympanoplasty, also called eardrum repair, refers to surgery performed to reconstruct a perforated tympanic membrane (eardrum) or the small bones of the middle ear. Eardrum perforation may result from chronic infection or, less commonly, from trauma to the eardrum.

Objective: The Objective of the study is to evaluate the surgical outcomes of tympanoplasties with and without mastoidectomies in terms of graft uptake and hearing improvement.

Methods: A total of 56 patients undergoing tympanoplasties with and without mastoidectomy were included and followed up for a period of one year.

Results: There was no significant difference in the surgical outcomes of both the surgeries in terms of graft uptake and hearing improvement. In unilateral cases Belfast rule of thumb application enables the actual hearing benefit of the patient.

Conclusion: There is no difference in related to outcome The addition of cortical mastoidectomy to tympanoplasty did not improve the outcome of surgeries done for mucosal type of chronic suppurative otitis media.

KEY WORDS: Cortical M astoidectomy, Tympanoplasty, Belfast Rule.

Address for correspondence: Dr. Hemendra Bamaniya, 3-N-20,Sector-5,Prabhat Nagar, Hiran M agri, Udaipur -313002, Rajasthan, India. E-Mail:drhemendra12345678@gmail.com

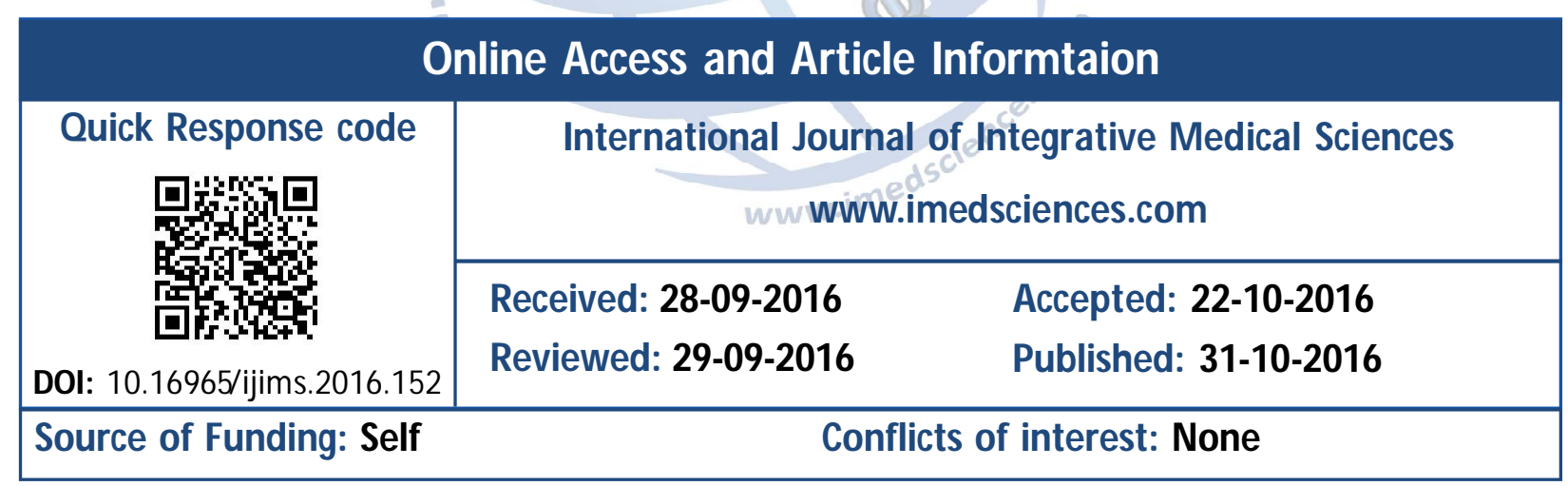

\section{INTRODUCTION}

Tympanoplasty, also called eardrum repair, refers to surgery performed to reconstruct a perforated tympanic membrane (eardrum) or the small bones of the middle ear. Eardrum perforation may result from chronic infection or, less commonly, from trauma to the eardrum.

The tympanic membrane of the ear is a threelayer structure. The outer and inner layers consist of epithelium cells. Perforations occur as a result of defects in the middle layer, which contains elastic collagen fibers. Small perfora- -tions usually heal spontaneously. However, if the defect is relatively large, or if there is a poor blood supply or an infection during the healing process, spontaneous repair may be hindered. Eardrums may also be perforated as a result of trauma, such as an object in the ear, a slap on the ear, or an explosion [1]. The purpose of tympanoplasty is to repair the perforated eardrum, and sometimes the middle ear bones that consist of the incus, malleus, and stapes. Tympanic membrane grafting may be required. If needed, grafts are usually taken from a vein 
or fascia (muscle sheath) tissue on the lobe of the ear [2]. Synthetic materials may be used if patients have had previous surgeries and have limited graft availability. The mastoid is a honeycomb cavity in the temporal bone, which lies directly behind the ear and is connected to the middle ear space. When a hole arises in the eardrum due to previous injury or infection, or when a long-standing infection persists with tissue in the middle ear or mastoid, mastoid surgery often becomes necessary to alleviate this infection. This part of the procedure is called mastoidectomy. Surgery for tubotympanic type of chronic suppurative otitis media is the commonest otological surgical procedure in our country [3].

This study discusses the various pre-operative factors which play a major role in the postoperative success of two various surgeries myringoplasty and cortical mastoidectomy with type 1 tympanoplasty. The hearing benefit is determined by Air bone gap closure and also subjective evaluation of hearing is done by applying Belfast rule of thumb.

\section{MATERIALS AND METHODS}

This Prospective study was conducted at RNT medical college and attached M.B hospital, Udaipur, Rajasthan from Dec 2009 to Dec 2011. Study Involves total 56 patients attending ENT OPD with- mucosal disease at Our hospital for a period of one year and the patients were followed up for a period of one year. Among them, 6 patients did not turn up for follow up and they were excluded from the study.

Inclusion criteria: Patients between 15 to 50 years of both sexes, after elimination of focal sepsis, having either wet or dry ears and pure tone audiometry showing conductive hearing loss.

Exclusion Criteria: Patients with congenital or acquired abnormalities of ear, unsafe ear, undergone ear surgeries previously and with pure tone audiometry showing mixed and sensorineural hearing loss were excluded from the study.

Methodology: M inimum duration of discharge for the patients under study was lyear and maximum was 15 years. $M$ inimum duration of hearing impairment was 6 months and maximum was 10 years. For all patients, under study with wet ears, ear swabs were taken from middle ear and for patients with culture positive results, treated with specific antibiotics prior to surgery.

All cases were admitted, pre-operative examination done under microscopy, subjected to endoscopic eustachian tube evaluation (those with normally looking pharyngeal end of eustachian tube orifice were taken up for study) and hearing assessment done with tuning fork tests and pure tone audiogram. All patients were informed about their need for a follow up period of one year. Informed written consent to undergo surgery was obtained from all patients. Mastoid shaving and local preparation was done in the ward prior to surgery. All cases were done under GA. Premedication and local infiltration was same for all cases.

Group A: About 25 patients with dry ear for more than 6 weeks and with only conductive hearing loss, were subjected to myringoplasty and considered as Group A. About 13 patients with unilateral disease and 12 patients with bilateral disease were taken up for study. For patients with bilateral disease, worse ear was taken up for surgery. All patients were taken up for surgery under GA. Trans canal approach was followed in 21 cases and in 4 cases with narrow external auditory canal post- aural approach was followed. 4 quadrant local infiltration was given with $2 \%$ xylocaine with adrenaline premixed solution. Temporalis fascia graft harvested by a separate incision over supra auricular region for patients done through trans-canal approach \& in patients done through post-aural approach, graft was harvested via the same incision. Temporalis fascia graft kept by underlay technique. All cases were followed up for 3,6 months and one year for graft uptake and postoperative hearing evaluations done at 3,6 months and one year. Results of hearing benefit, compared with pre-op and post-op air bone gap and for unilateral disease Belfast rule of thumb applied.

Group B: About 25 patients with wet ear and with no active infection as per microbiological report $\&$ with conductive hearing loss were subjected to cortical mastoidectomy with type 1 
tympanoplasty and considered as Group B. About 15 patients with unilateral disease and 10 patients with bilateral disease were taken up for study. For patients with bilateral disease, worse ear was taken up for surgery.

All patients were taken up for surgery under GA. Post-aural approach was followed in all cases. Post aural incision made, temporalis fascia graft harvested through the superior aspect of the incision as the first step, incision deepened and mastoid cortex exposed. Pinna retracted anteriorly, incision made in the posterior canal wall skin from 6 o'clock to 12 o'clock position and tympanomeatal flap was elevated. Granulations if present in the tympanum were removed, mobility of ossicular chain were checked. Mastoid cortex was drilled out in all cases, patency established. For about 21 patients granulations noted in antrum removed and in 4 patients antrum found free of disease. For all patients, temporalis fascia graft kept by underlay technique, tympanomeatal flap repositioned and post-aural wound closed in layers. All cases were followed up for 2, 6 months and 1 year for graft uptake and postoperative hearing evaluations done at 3,6 months and 1 year. Results of hearing benefit, compared with pre-op and post- op air bone gap and for unilateral disease, Belfast rule of thumb applied.

Even though socio-economic status does influence the disease, all the patients attending RNT M edical College and M B Hospital Hospital belongs to lower socio-economic group. Hence this parameter is not included in this study.

\section{RESULTS}

Total number of cases registered in this study was 56 patients, who came to the ENT Department with M ucosal disease. Among them 6 patients did not turn up for follow up and hence they were not included in the study.

The overall graft take up rate in both the surgeries was $88.33 \%$. The overall hearing benefit was $90 \%$, excellent with $<10 \mathrm{~dB} A B G$ in $51.67 \%$ and Good with $<20 \mathrm{~dB}$ in $38.33 \%$. There was no postoperative complications, deterioration in hearing or sensorineural hearing loss in all 50 patients.
In Group A , 15 were females \& 10 were males, 13 patients had unilateral and 12 had bilateral disease, 20 had medium sized central perforation and 05 had subtotal perforation, 10 had good pre-op Air bone gap of 10 to $20 \mathrm{dBHL} 15$ patients had a fair pre-op Air bone gap of 20 to $30 \mathrm{dBHL}$.

1. Overall graft take up rate was $86.6 \%$.

2. Otoscopic evaluation of 20 patients with medium sized perforation at the end of 2 months, revealed 15 to be intact, 5 residual perforation in the antero - inferior quadrant. In 5 patients with subtotal perforation, 3 were intact, 1 with residual perforation $\& 1$ grafts got rejected because of post-op wound infection. Otoscopic examination at the end of 6 months $\& 1$ year revealed - for all 20 patients with medium size perforation, tympanic membrane was intact and out of 5 patients with subtotal 2 were with residual perforation $\& 1$ with rejection of graft. Hence the size of the perforation does have a role in graft take up rate. This is statistically significant by applying chi square test with $p$ value $<0.05$.

3. In 10 patients with good pre-opABG, the postop ABG was excellent. In 15 patients with fair pre-op $A B G$, the post-op $A B G$ results were, excellent in 9, good in 8 and fair in 3 patients accounting for $46.66 \%$ in excellent, $40 \%$ in good and $13 \%$ in fair groups. By applying Chi square test, these results are statistically significant Hence, for the patients with lesser pre-operative Air bone gap have a better post-operative hearing.

4. When Belfast rule of thumb was applied to 13 patients with unilateral disease, 10 of them with medium sized perforation, felt subjectively better and out of 3 of them with subtotal perforation, 2 felt better $\& 1$ patient felt hearing same as the pre operative status. These results are statistically significant with $p$ value $<0.05$, implies that Belfast rule of thumb interpret the post-operative hearing benefit in a better way than the Air bone gap ,which tells about only the technical success.

5. The correlation coefficient between the duration of discharge and the pre-op $A B G$ is 0.4172 and that between the duration of hearing impairment with pre-op ABG is 0.3821 and is statistically significant. The correlation coeffici- 
-ent between the duration of discharge and the post-op ABG is 0.4544 and that between the duration of hearing impairment with post-op ABG is 0.4489 and is statistically significant, implies that the patients with lesser duration of discharge and hearing impairment had better post-operative hearing than the patients with longer duration of disease and hearing impairment.

6. The other parameters, such as age, sex, weight does not have influence on the outcome of results in this study.

7. The type of approach does not have a significant $p$ value with graft intake in this study. Applying chi square test, the Pearson value is 0 .

In Group B, 17 were females \& 8 were males, 15 patients had unilateral and 10 had bilateral disease. 18 had medium central perforation and 07 had subtotal perforation, 08 had good preop Air bone gap of 10 to $20 \mathrm{dBHL} 17$ patients had a fair pre-op Air bone gap of 20 to $30 \mathrm{dBHL}$.

1. Overall graft take up rate was $90 \%$.

2. The relationship between Graft take up rate and size of the perforation is statistically significant by applying chi square test with person value of 0.02535 .

Otoscopic evaluation of 15 patients with medium sized perforation at the end of 2 months, revealed 14 to be intact, 1 residual perforation in the antero-inferior quadrant. In 07 patients with subtotal perforation, 05 were intact, 2 with residual perforation. Otoscopic examination at the end of 6 months $\& 1$ year revealed - for all 18 patients with medium size perforation, tympanic membrane was intact and out of 07 patients with subtotal 3 were with residual perforation. Hence the size of the perforation do have a role in graft take up rate even when cortical mastoidectomy is done along with repair of tympanic membrane.

3. In this study, out of 18 patients with medium sized perforation, 8 had a good \& 17 had fair pre-op ABG. Out of 07 patients with subtotal perforation, 6 patient had good $\& 01$ had fair pre-op ABG, implies that $85.71 \%$ of the patients with subtotal perforation had pre-op ABG of 20 to $30 \mathrm{~dB}$.

4. Out of 25 patients, included in this procedure -re, $50 \%$ had excellent, $50 \%$ had good \& $10 \%$ had fair post-op ABG. Those with medium sized perforation had better results than those with subtotal perforation.

5. On comparison of pre-op $A B G \&$ post-op ABG, all 8 patients with good pre-op ABG improved to excellent, out of 17 with fair $A B G, 3$ were excellent, 12 were good and 2 were fair postoperatively. This result is highly statistically significant with pearson value of 0.00006 .

6. Graft intake in 4 patients with disease free antrum mucosa is $100 \%$.

7. Out of 25 patients, 21 patients had disease in the antrum \& 4 had a healthy antrum. $77.85 \%$ of the patients with healthy antrum and $9.5 \%$ of the patients with diseased antrum had good preop Air bone gap $90.5 \%$ with diseased and $22.2 \%$ with healthy antrum had fair preop Air bone gap.

8. In patients with healthy antrum, the post-op ABG was excellent in $88.9 \%$ and $66.7 \%$ of the patients

with diseased antrum had a good ABG postoperatively.

9. On applying Belfast rule of thumb to 15 patients with unilateral disease, $83.3 \%$ had better hearing, implies the post-operative hearing benefit better than assessment with Air bone gap.

10. The correlation coefficient between the duration of discharge and the pre-op ABG is 0.3803 and that between the duration of hearing impairment with pre-op ABG is 0.3776 . The correlation coefficient between the duration of discharge and the post-op ABG is 0.3794 and that between the duration of hearing impairment with pre-op ABG is 0.3729 and are statistically significant, implies that the patients with lesser duration of discharge and hearing impairment had better post-operative hearing than the patients with longer duration of disease and hearing impairment.

11. The other parameters, such as age, sex, weight does not have influence on the outcome of results in this study.

\section{DISCUSSION}

Analysis of 50 cases undergone surgery for tubotympanic disease is presented here and the 
same is compared with similar and related studies available in literature.

1. Graft Take Up Rate: The overall graft take up rate in Myringoplasty in this study was $86.6 \%$, which is within the range to the studies available. The graft take up rate in various studies were [Table 1].

Table 1: Graft take up rate [1].

\begin{tabular}{|c|c|c|c|}
\hline Study & No of cases & year & $\begin{array}{c}\text { Graft take up } \\
\text { rate }\end{array}$ \\
\hline Gibb, Chang et al [5] & 365 & 1982 & $91.40 \%$ \\
\hline Black, PJ Wormald [3] & 261 & 1995 & $78 \%$ \\
\hline Raj A Vidit [1] & 50 & 1999 & $84 \%$ \\
\hline Kotecha et al [7] & 107 & 1999 & $82.20 \%$ \\
\hline Yasuo, Mishiro et al [1] & 104 & 2001 & $94.40 \%$ \\
\hline Kageyama et al [6] & 290 & 2001 & $82.10 \%$ \\
\hline Alberrra et al [2] & 85 & 2006 & $86 \%$ \\
\hline
\end{tabular}

The graft take up rate in Cortical mastoidectomy with type 1 tympanoplasty is $90 \%$ which is also within the range to the studies available in literature [Table 2].

Table 2: Graft take up rate [2].

\begin{tabular}{|c|c|c|}
\hline Studies & No of cases & Graft take up rate \\
\hline Yasuo, Mishiro et al [1] & 147 & $90 \%$ \\
\hline Adnan saleem et al [2] & 85 & $92.95 \%$ \\
\hline McGrew et al [2] & 100 & $91 \%$ \\
\hline Rehl CM et al [2] & 135 & $90.40 \%$ \\
\hline
\end{tabular}

2. Graft Failure Rate: Graft failure rate in various studies were [Table 3].

Table 3: Re-perforation Rate \% [1].

\begin{tabular}{|c|c|}
\hline Studies & Re-perforation Rate \% \\
\hline Alberra et al [2] & 7 to 21 \\
\hline Vartianien et al [14] & 10.6 \\
\hline Adnan, saleem et al [2] & 7.05 \\
\hline
\end{tabular}

In this study, in myringoplasty series the rate of residual perforation is $6.7 \%$ and the rate of rejection of graft is $6.7 \%$. The probable cause of residual perforation is the slippage of the graft from its position and the cause for rejection is the post operative infection of the operated site, which is same as the reason cited by the study of Vartianien et al and Kotecha et al.[7,14] Study of Alberra et al also includes the efficiency of the surgeon and the surgical techniques [2].

3. Pre-operative Hearing: In this study the pre operative pure tone average in $\mathrm{M}$ yringoplasty series was between 30 to $40 \mathrm{~dB}$ with average being $38 \mathrm{~dB}$ and in Cortical mastoidectomy with type 1 tympanoplasty series between 30 to 55 $\mathrm{dB}$ with average being $42.16 \mathrm{~dB}$. As the perforation size increases, the preoperative hearing threshold also increases - this is consistent with the study conducted by M ehta et al, Bhusal et al Nepal A Bhandary et al, Kageyama etal, Aiberra et al and Saeed et al.[1,2]

4. Post Operative Hearing Benefit: Hearing improvement in various studies quoted in literature were [Table 4];

Table 4: Hearing Improvement [2,3].

\begin{tabular}{|c|c|}
\hline Study & $\begin{array}{c}\text { Hearing } \\
\text { Improvement }\end{array}$ \\
\hline Adnan et al [2] & $85.88 \%$ \\
\hline Rizer et al [2,3] & $84.90 \%$ \\
\hline Raj A Vidit et al [2] & $68 \%$ \\
\hline Kotecha et al [7] & $67 \%$ \\
\hline
\end{tabular}

In this study, the overall post operative hearing benefit based on Air bone gap was $90 \%$ with $<10 \mathrm{Db}$ in $51.67 \%$ and $<20 \mathrm{db}$ in $38.33 \%$. As hearing benefit is better assessed with the subjective evaluation, in this study we have applied Belfast rule of thumb in patients with unilateral disease. In 23 patients with unilateral disease in M yringoplasty series, $95.7 \%$ felt significant improvement in hearing and $4.3 \%$ felt no improvement. In 18 patients with unilateral disease in Mastoidectomy with type 1 tympanoplasty series, $83.3 \%$ felt significant improvement in hearing and $16.7 \%$ felt no improvement.

\section{CONCLUSION}

From Our study we Would like to conclude that the success of $M$ yringoplasty in terms of graft uptake and hearing improvement is better in patients with lesser duration of disease, less preoperative Air bone gap and with medium sized perforations when compared to subtotal perforations. The success of Cortical mastoidectomy with type tympanoplasty in terms of graft uptake and hearing improvement is better in patients with lesser duration of disease and less pre-operative Air bone gap.

\section{REFERENCES}

[1]. Downey, T. J., A. L. Champeaux, and A. B. Silva. AlloDerm Tympanoplasty of Tympanic Membrane Perforations. American Journal of Otolaryngology. (January/February 2003;24:6-13. 
[2]. Albera R, Ferrero V, Canale A. Tympanic reperforation in M yringoplasty: Evaluation of prognostic factors. Ann Otol Rhinol Laryngol. 2006;115(12):875-9.

[3]. Black JH, Wormald PJ Myringoplasty - Effects on Hearing and contributing factors. South Afr M edi Jour 1995;85(1):41-3.

[4]. Uzun, C., M. Velepic, D. Manestar, D. Bonifacic, and T. Braut. "Cartilage Palisade Tympanoplasty, Diving and Eustachian Tube Function." Otology \& Neurotology 24 (M arch 2003):350-1.

[5]. Gibb AG, Chang SK et al. M yringoplasty ( A review of 365 operations). J Laryngology Otology 1982;96(10):915-30.

[6]. Kageyama-Escobar AM, Rivera- M oreno MA, RiveraM endez A. Risk factors for M yringoplasty failure. Gac M ed Mex. 2001 May-June;137(3):209-20.

[7]. Kotecha B, Fowler S, Topham J M yringoplasty: A prospective Audit Study. Clin Otolaryngol Allied Sci. 1999 Apr;24(2):126-9.

[8]. Uzun, C., M. Velepic, D. M anestar, D. Bonifacic, and T. Braut. Cartilage Palisade Tympanoplasty, Diving and Eustachian Tube Function. Otology \& Neurotology. March 2003;24:350-1.
[9]. M irkoTos Manual of Middle ear surgery- volume 1. [10].M ishiro Y, Sakagami M, Takahashi Y, Kitahara T, Kijikawa H. Tympanoplasty with and without Mastoidectomy in non cholesteatomatous chronic otitis media. Eur Arch Otorhinolaryngol. 2001;258(1):13-15.

[11]. Sheahan, P., T. O'Dwyer, and A. Blayney. Results of Type 1 Tympanoplasty in Children and Parental Perceptions of Outcome of Surgery. Journal of Laryngology \& Otology. June 2002;116:430-4.

[12].Scott-Brown Otorhinolaryngology, Head and Neck Surgery 6th edition.

[13]. Scott-Brown Otorhinolaryngology, Head and Neck Surgery 7th edition.

[14].Vartiainen E, Karga J, Karjalainen S,Harma R.Failures in Myringoplasty. Archives Otolaryngol. 1985;242(1)27-33.

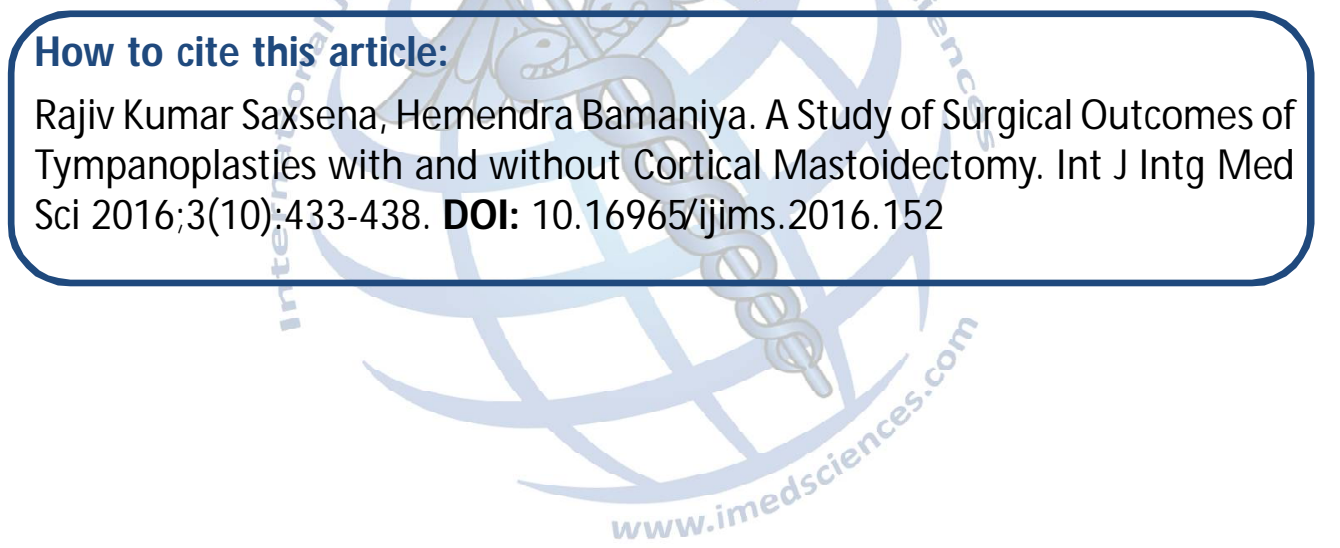

